\title{
Les exigences d'essais pour une qualification matérielle des équipements classés sureté nucléaire
}

\author{
Frédérick BORDES ${ }^{a}$ \\ AREVA NP, 1 Place Jean Millier, 92084 Paris - La Défense
}

\begin{abstract}
: $13.5 \%$ of the worldwide electricity is produced by 438 nuclear reactors in operation. 71 are under construction. In the nuclear world, the highest safety level has always constituted an absolute priority: safety of products, safety of the solutions developed for the benefit of the customers, and safety of operations. To achieve these objectives, a component, installed in a nuclear power plant, which contributes to a safety function, is safety classified and has to be qualified accordingly. The qualification consists in demonstrating whether by tests or by analysis that the equipment is able to work in the normal environment of the plant and in degraded conditions based on accidental event analyses. The tests sequence can include electrical tests, EMC tests, climatic/environment tests, mechanical tests, seismic tests, irradiation tests. As a matter of fact, a qualification lasts between 2 to 3 years including the different steps from the design to the final qualification documents. The aim of this presentation is to give an overview of the equipment qualification in the nuclear domain and related requirements to perform the test sequences. 3 topics will be shared: The activities of the qualification in the nuclear domain, the test requirements (test severities, test facilities...) and the quality requirements (traceability, IEC standards, documentation, accreditation...)
\end{abstract}

\section{Introduction}

La sureté nucléaire définit l'ensemble des activités ayant trait au maintien de l'intégrité des mécanismes, processus, outils ou instruments contenant de la matière radioactive, permettant de garantir l'absence d'effets dommageables sur les individus et l'environnement.

Une notion de classification d'équipements apparait alors selon les fonctions de sureté que doit remplir l'équipement dans une centrale nucléaire, en fonctionnement normal ou en conditions accidentelles. Une démonstration doit être fournie sur l'aptitude des équipements à respecter ces niveaux de classification.

La qualification des équipements consiste à prouver que le matériel est apte à assurer le service requis dans les conditions d'environnement correspondant aux conditions de fonctionnement normales ou accidentelles dans lesquelles il peut se trouver.

\section{Les activités de Qualification dans le domaine nucléaire}

\subsection{Classification des équipements}

A l'échelle mondiale, la classification des équipements n'est pas standardisée. Par exemple, les Etats-Unis font uniquement une séparation entre les équipements classés servant au fonctionnement normal d'une centrale et ceux utilisés en cas d'accident. En France, les équipements sont classés selon leur niveau d'exigence pour la sureté nucléaire. La qualification distingue :
- Les matériels de catégorie K1, situés à l'intérieur du bâtiment réacteur, ayant à assurer leurs fonctions dans des conditions

d'environnement correspondant aux conditions de fonctionnement normales, accidentelles et/ou post-accidentelles, et sous sollicitations sismiques.

- Les matériels de catégorie Accident Grave (AG), situés à l'intérieur du bâtiment réacteur, ayant à assurer leurs fonctions dans des conditions d'environnement correspondant aux conditions de fonctionnement normales, accidentelles et/ou post-accidentelles du cœur du réacteur, et sous sollicitations sismiques.

- Les matériels de la catégorie K2, situés à l'intérieur du bâtiment réacteur, ayant à assurer leurs fonctions dans des conditions d'environnement correspondant aux conditions de fonctionnement normales et sous sollicitations sismiques.

- Les matériels de la catégorie K3, situés hors du bâtiment réacteur ayant à assurer leurs fonctions dans des conditions d'environnement correspondant aux conditions de fonctionnement normales, et sous sollicitations sismiques.

- Les matériels de la catégorie K3ad, situés hors du bâtiment réacteur ayant à assurer leurs fonctions dans des conditions d'environnement correspondant aux conditions de fonctionnement en ambiance dégradée (pression, température, irradiation,..), et sous sollicitations sismiques.

\footnotetext{
a Email de correspondance de l'auteur : frederick.bordes@areva.com
} 


\subsection{La stratégie de qualification}

Toute la démarche consiste à déterminer une stratégie de démonstration pouvant être faite soit par analyse, soit par essais, ou soit par méthode combinée (analyse + essais).

Dans le cas des essais, une liste et l'ordre des tests sont définis en notifiant les sévérités à appliquer et les critères d'acceptation.

La finalité de la qualification est d'apporter la preuve que l'équipement répond à tous les exigences demandées selon sa classification, et selon les conditions d'ambiance et environnementales.

\section{Les exigences d'essais}

La norme CEI 60780 [1] définit d'une manière générale la qualification du matériel électrique de sureté. De plus, des référentiels constructeurs [2], [3], définissent et décrivent les essais à réaliser pour une qualification ainsi que les documents associés.

\subsection{La séquence d'essais}

La démonstration de l'aptitude d'un équipement à fonctionner dans les conditions pour lesquelles il a été prévu, est basée sur une stratégie de qualification.

Dans le cas des essais, il est important de respecter la séquence définie (ordre et critères). Plusieurs types d'essais sont réalisés :

- Les essais de référence,

- Les essais aux limites d'emploi

- fonctionnelles, Les essais de robustesse dans

- le temps, Les essais accidentels.

\subsection{Les essais de référence (initiaux et finaux)}

Des essais de référence sont réalisés au début et à la fin de la séquence d'essais. Cela peut comprendre :

- Des examens visuels

- Des essais électriques

- Des essais fonctionnels

Ces essais servent de référence pendant la qualification. Des essais fonctionnels intermédiaires sont, de plus, réalisés au cours de la séquence pour vérifier le bon fonctionnement de l'équipement.

\subsection{Les essais aux limites d'emploi fonctionnelles}

L'équipement est également testé dans sa limite de fonctionnement (fonctionnellement, électriquement, environnementalement). Généralement et selon l'équipement, les essais réalisés sont :

- Variation de tension

- Essais climatiques (entre $-70^{\circ} \mathrm{C}$ et $+55^{\circ} \mathrm{C}$ )

- Humidité

- CEM (Compatibilité Électromagnétique)

\subsection{Les essais de robustesse dans le temps}

Les essais réalisés se basent sur une hypothèse de durée de vie qualifiée qui dépend de l'équipement et qui est comprise entre 10 ans et 60 ans. Cette durée de vie qualifiée ne peut être assurée si la séquence n'est pas scrupuleusement suivie, en particulier pour ce qui concerne les sévérités et l'ordre des essais.

Le matériel subit une série de tests simulant le vieillissement et doit montrer sa robustesse fonctionnelle et mécanique dans le temps :

- Essais climatiques (variations de température, chaud, froid, humidité, ...),

- Essais mécaniques (vibrations, fonctionnement prolongé, ..),

- Essais irradiation pour les équipements classés $\mathrm{K} 1$ et $\mathrm{K} 2$.

\subsection{Les essais accidentels}

\subsubsection{Le séisme}

La norme CEI 60980 [4] recommande les pratiques pour la qualification sismique. L'essai séisme est, dans la majorité des cas, réalisé sur du matériel « vieilli » par la séquence d'essais précédente (\$3.4). L'équipement est monté sur une table vibrante et subit des accélérations suivant un spectre spécifique selon sa localisation dans la centrale.

\subsubsection{L'irradiation accidentelle}

Selon sa localisation dans la centrale et sa fonction, l'équipement peut subir des doses d'irradiation et doit continuer à fonctionner. Des essais fonctionnels sont réalisés après un essai d'irradiation

\subsubsection{L'accident thermodynamique}

En cas d'accident thermodynamique (par exemple rupture canalisation vapeur), l'équipement doit résister à un profil Pression/Température particulier (par exemple $156^{\circ} \mathrm{C}$ et 5,5 Bars pour une qualification $\mathrm{K} 1$ ).

\section{Les exigences Qualité}

Le domaine nucléaire impose une rigueur dans la traçabilité de la conception jusqu'à l'exploitation a mise en opération sur site afin de pouvoir démontrer à tout moment le respect des exigences demandées.

Certains documents sont analysés par les autorités de sureté nucléaire de chaque pays utilisant l’énergie électronucléaire. 


\subsection{Traçabilité}

Toutes les étapes lors d'une qualification doivent être tracées afin de garantir le respect des exigences demandées. Cela impose un suivi durant les phases de :

- Conception (spécifications),

- Essais/Qualification (fabrication du spécimen d'essais, suivi des essais, validation de la qualification),

- Fabrication série (fabrication à l'identique du spécimen d'essais),

- Installation (suivi essais sur site) En opération

- (essais périodiques, maintenance, ...).

Toute évolution et toute anomalie doivent être tracées et validées afin de garantir la qualification et son maintien durant la vie de la centrale nucléaire.

\subsubsection{Spécimen de qualification}

Le spécimen de qualification doit être représentatif des équipements montés sur site. Il doit être parfaitement décrit afin de connaître exactement les éléments qui seront qualifiés. De plus, un suivi de sa fabrication doit être réalisé afin de pouvoir fabriquer à l'identique les matériels série. La fabrication est tracée dans un dossier appelé « Dossier de référence $"$.

Le spécimen ne subit aucune modification pendant la phase de qualification (sauf cas particuliers qui doivent être tracés) et inclut, en général, 3 exemplaires de chaque composant à qualifier.

\subsubsection{Essais et accréditation}

Les essais sont basés sur des normes internationales, des codes et standards.

Un niveau d'exigence est demandé pour les essais de qualification. Les essais doivent être maîtrisés avec une gestion d'un système Qualité irréprochable. C'est pourquoi il est demandé de réaliser les essais sous une accréditation COFRAC (ou équivalent à l'ISO 17025).

Un programme de qualification définit la stratégie pour démontrer que l'équipement répond aux exigences demandées. Chaque test doit être parfaitement décrit (performances et critères à respecter, conditions environnementales, ...). Une séquence d'essais peut durer entre 6 mois et 18 mois selon la classification du matériel testé.

Un non-respect des critères définis entraîne la gestion d'une non-conformité.

\subsubsection{Gestion des non-conformités}

Lors des essais, des aléas peuvent apparaître. Dans ce cas, il est demandé de ne pas poursuivre les essais tant qu'une analyse des causes ne permette pas de comprendre l'origine et les raisons de la défaillance. Une fiche de non-conformité est alors ouverte incluant ainsi l'analyse et les actions associées pour clore cet aléa.
Toute non-conformité est tracée et incluse dans le dossier de qualification pour apporter toute la transparence et la démonstration de la qualification.

Selon l'impact de la non-conformité, les essais peuvent être soit repris, soit poursuivis, soit même complétement stoppés car une nouvelle conception est alors nécessaire. Cela entraîne dans ce cas, une nouvelle qualification.

\section{Références}

[1] CEI 60780 - 1998 : Centrales nucléaires Equipements électriques de sûreté Qualification.

[2] RCC-E : Règles de conception et de construction des matériels électriques des îlots nucléaires.

[3] RCC-M : Règles de conception et de construction des matériels mécaniques des îlots nucléaires.

[4] CEI 60980 - 1989 : Pratiques recommandées pour la qualification sismique du matériel électrique du système de sureté dans les centrales

électronucléaires 